\title{
Layouts and Cells in Integral Photography and Point Light Source Model
}

\author{
Vladimir V. Saveljev* \\ Research Institute of Electrical and Computer Engineering, Hanyang University, Seoul 133-791, Korea \\ Seung-Jung Shin \\ Department of Information Technology, Hansei University, Seoul 435-742, Korea
}

(Received December 9, 2008 : revised February 24, 2009 : accepted March 6, 2009)

\begin{abstract}
The similarity between two groups of displaying methods is demonstrated in two ways, analytically and experimentally. A variety of layouts of the integral photography and display devices based on the point light source model is classified and analyzed in terms of projections and common/ separate image planes. In particularly, the transformation matrix is found. Simulation experiments based on the image processing were performed. The layouts, analytical formulas, and experimental results show the similarity of both groups for several layouts.
\end{abstract}

Keywords: 3-D display, Integral photography, Integral imaging, Multi-view imaging, Autostereoscopic display, Full-parallax display

OCIS codes : (100.0100) Image processing; (110.0110) Imaging systems; (110.6880) Three-dimensional image acquisition; (100.6890) Three-dimensional image processing; (120.2040) Displays

\section{INTRODUCTION}

There are two big groups of autostereoscopic 3D displaying methods, the integral photography and 3D display systems based on the point light source model. Previously, a comparison between 3D image forming methods was made [1] basing on the viewing zone analysis. In the current article, we provide geometrical and experimental comparison of these groups of methods because these two seem to be the most representative and the most developed among 3D imaging methods [2].

The integral photography (IP) which is often referred to as the integral imaging is based on a multi-lens photographic image obtained with using a lens array [3] which is used in both stages, capturing and displaying. The lens array is one of the most essential parts of IP equipment.

A 3D display system based on the point light source model (PLS) [4] can be also based on simultaneous photographs taken by several cameras at once. A possible layout of cameras however is not as strictly limited as

*Corresponding author: saveljev@hanyang.ac.kr that of the uniform arrangement of lenses in a lens array. In displaying, various optical plates can be used, lens arrays, lenticular, or parallax barrier.

The similarity/dissimilarity will be tested in two ways, analytically for various layouts and numerically by processing of image cells in simulation experiments.

We will consider several geometric layouts which allow us to determine an interrelation between IP and PLS, two groups to display full-parallax 3D images. Therefore in this article, we do not include any time related issues as in [1] but rather consider more geometry. A variety of layouts including parallel/radial, orthogonal/nonorthogonal (oblique) will be classified and analyzed in terms of projections and common/separate image planes. For layouts we will find an analytical description including the transformation matrix.

The image processing applied to images is essentially cellular (based on image cells). Two examples of similar processing can be found in $[5,6]$. In particularly, cells on the infinite height are actually shown in Fig. 3 in [5]. Fig. 2 in [6] (modified elemental images) gives another example of what is called interlace in image processing. 
However in our case, the image processing is two-dimensional. By comparing results obtained after a processing method taken from one group was applied to another group, one can find experimentally whether a similarity/ dissimilarity between the pixel layouts within cell of two 3D imaging groups exists or not.

The analytical approach combined with experimental results will allow us to make a direct confirmation of similarity in question and to find in which cases this similarity exists.

\section{MATHEMATICAL BACKGROUND}

The IP image consists of many elementary images obtained under each elementary lens of a lens array [3]. This looks like a simultaneous photograph taken by a camera array. The image plane (film or digital sensor) is located at the focal distance $z_{0}$ under the lens array or $z_{0}$ behind a pinhole camera. An area beneath each lens can be considered as a cell of the whole image. Geometrically important is that the lenses can not be controlled individually but are arranged uniformly in one plane with parallel axes.

The PLS image also includes images taken by a (virtual) camera array [4]. These view images are split into small parts (e.g., pixels) and rearranged into a single cellular image consisting of image cells. This arrangement is based on relative locations of cameras. The period of the resulting cells should correspond to the period of optical plate used in displaying.

Here we describe the camera array through a projection transformation which can be conveniently represented in the homogeneous coordinates [7]. In the matrix form [8] it looks like follows

$$
X^{\prime}=T \cdot X
$$

where $X$ and $X^{\prime}$ are the original and resulting homogeneous 4 -vectors and $T$ is the homogeneous transformation matrix $4 \times 4$.

To describe a projection transformation performed by a camera at $\left(x_{c}, z_{c}\right)$ located in front of a image plane $\mathrm{z}=0$ as shown in Fig. 1.

we can use the following matrix

$$
T=\left(\begin{array}{cccc}
1 & 0 & -x_{c} / z_{c} & 0 \\
0 & 1 & 0 & 0 \\
0 & 0 & 1 & 0 \\
0 & 0 & -1 / z_{c} & 1
\end{array}\right)
$$

The corresponding transformation in the regular coordinates look as follows

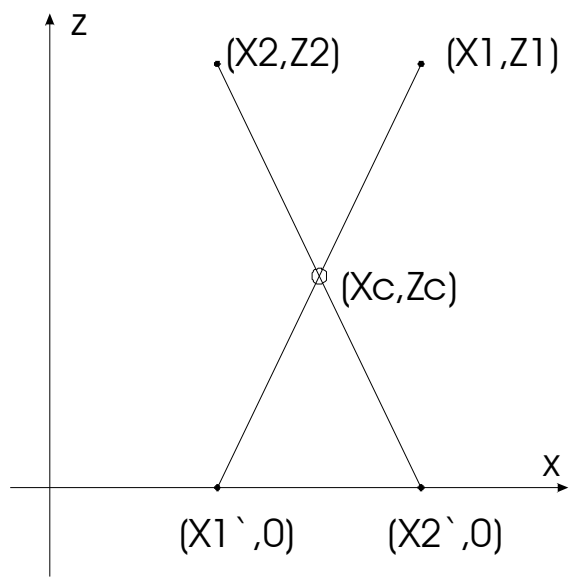

FIG. 1. Camera at $\left(x_{c}, z_{c}\right)$.

$$
x^{\prime}=\frac{x-\frac{x_{c}}{z_{c}} z}{1-\frac{z}{z_{c}}}
$$

\section{CAMERA LAYOUTS}

In a lens array the effective cameras are located at the centers of lenses and therefore are displaced uniformly along x-axis; the coordinates of the centers of the lenses (effectively cameras) are

$$
\begin{aligned}
& x_{c i}=i D, i=0, \ldots, N-1 \\
& z_{c i}=z_{0}
\end{aligned}
$$

where $i$ is the current number of the camera, $D$ is the distance between the elementary lenses (their period), $N$ is the total amount of cameras along x-axis, $z_{0}$ is the distance to the image plane (an effective focal length for a pinhole camera) which are supposed to be equal for all cameras.

For a lens array, the following expression for the image of $i$-th camera can be derived from (3),

$$
x^{\prime}(i)=\frac{x-\frac{i D}{z_{0}} z}{1-\frac{z}{z_{0}}}
$$

The layouts principally can be applied to both, capturing and displaying stages excepting a few special cases like Section III.4.

\section{Parallel layout}

Here we will find an analytical description of layouts. Consider the points in the image plane equally displaced from the centers of camera images and find which parts 


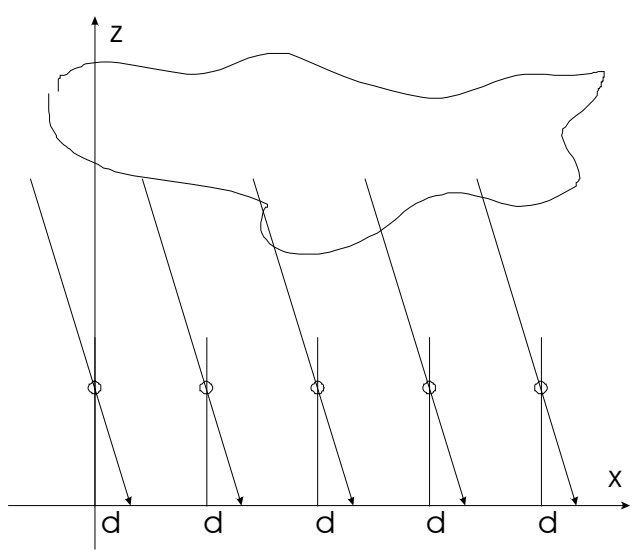

FIG. 2. Mapping an object to points equally displaced in all camera images.

of a photographed object are projected to there. For these points

$$
x^{\prime}(i)=i D+d
$$

where $d$ is the displacement, equal for all camera images.

In this case, the formula for $i$-th camera image (5) can be rewritten as follows

$$
\begin{gathered}
i D+d=\frac{x-\frac{i D}{z_{0}} z}{1-\frac{z}{z_{0}}} \\
x+\frac{d}{z_{0}} z-(i D+d)=0
\end{gathered}
$$

The above expression (8) represents the equation of a straight line having the slope (i.e. tangent) $-\frac{z_{0}}{d}$ and displaced by $i D+d$ along the x-axis. For a variety of integer values of $i$ it actually represents a family of parallel lines. This means that projected to the points equally displaced (in all camera images) are the points of the object located at the lines going from the object through the lens centers with the slope $-\frac{z_{0}}{d}$ as shown in Fig. 2, where the effective cameras are indicated by small circles.

\section{Parallel orthogonal layout}

In particularly, for the axial image $\mathrm{d}$ is equal to zero (and the configuration is orthogonal) so as

$$
x=i D
$$

The expression (9) matches the expression for lens coordinates (6) in case of an axial image and therefore means that $\mathrm{x}$-coordinate of the point of an object which

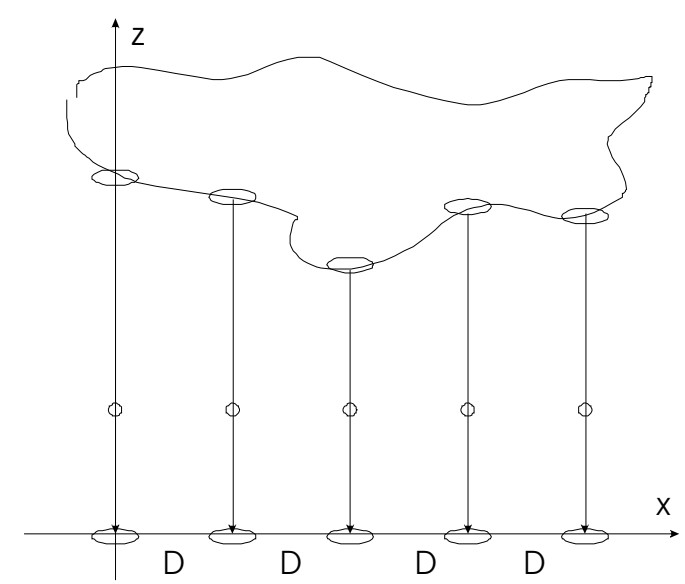

FIG. 3. Orthogonal mapping to axial points of images.

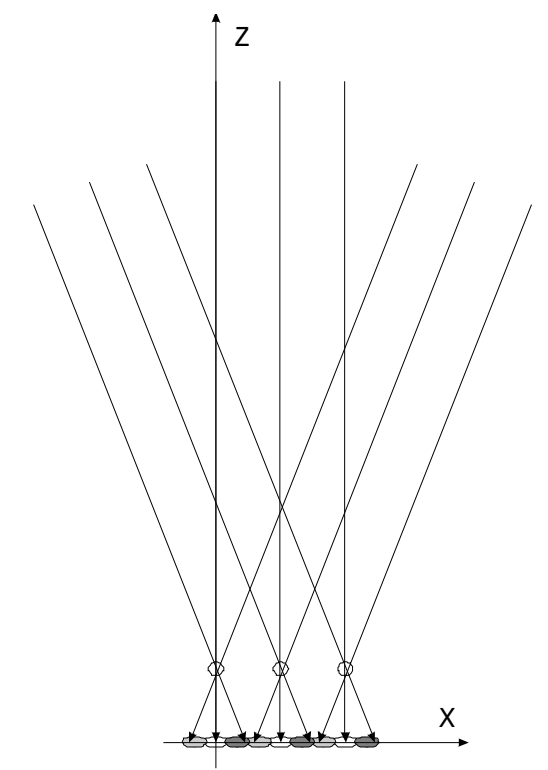

FIG. 4. Summarized discrete layout IP, common image plane.

is projected to the center of a lens image. It is the point of the object which is opposite to the lens center.

This is illustrated in Fig. 3. In this particular case, the picture of lines connecting projected points of the object and their projections look very similar to that of the orthogonal projection of an object to the plane $z=0$, especially if we would consider some areas instead of mathematical points.

This layout can be treated as a discontinuous, discrete projection which only includes a finite number of rays. Of course, in order to represent an object adequately, the period of projection rays should not be greater than the smallest period of the object's shape curve. Otherwise some essential parts of the object could be lost.

\section{Combined parallel layout}

A summarized picture of the discrete projection rays 


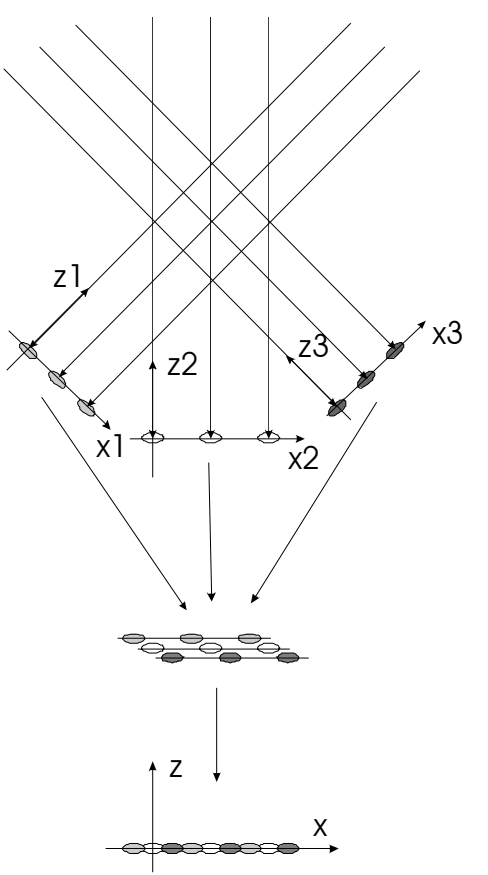

FIG. 5. Mapping an object to points equally displaced in all camera images.

found above is shown in Fig. 4 for 3 lenses and 3 slopes (including the central rays) corresponding to several displacements. The families of lines having the same displacement in the image plane differ in slope.

This picture of several images of displaced cameras looks similar to several non-orthogonal (but still parallel) projections to the plane $z=0$. Additionally, if the rays would be extended through the original image plane, the projection stage can be effectively isolated and all partial projections can be made orthogonal.

To do that, we get partial orthogonal projections to auxiliary perpendicular planes independently for each family of lines. Then we stack these partial projections with proper re-scaling (depending on their slope). With that, $x$-displacement of the local systems is incremented by the same value for each next partial projection so that the sum of increments is less than $D$. After that we put the stacked projections together onto the plane $z=0$. The procedure is shown in Fig. 5 . The resulting image is the same as in Fig. 4, therefore we can consider this layout as an equivalent of IP.

\section{Radial layout}

The picture of discrete orthogonal layout looks very similar to so called radial layout of cameras used sometimes in PLS. This confirms the similarity between IP and PLS. At the same time there exists a difference in angles of projection rays descending to the auxiliary planes.

In case of IP (Fig. 5) all the rays in a family are perpendicular to the corresponding auxiliary plane. Each partial projection is an orthogonal projection which cor-

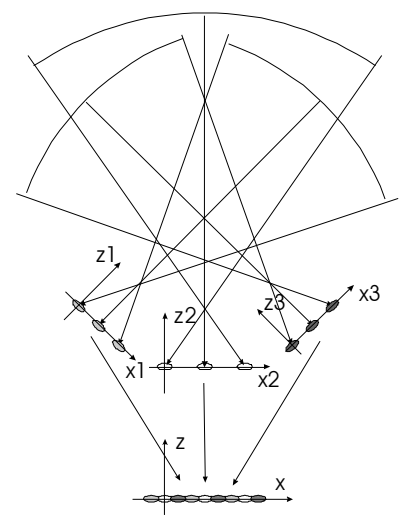

(a)

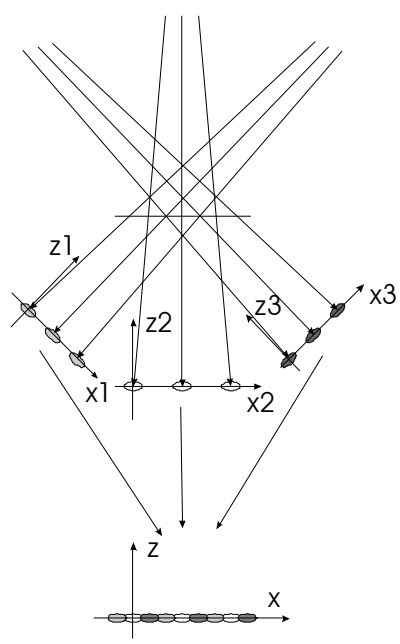

(b)
FIG. 6. Radial layouts PLS differing in focal distance, separate image planes.

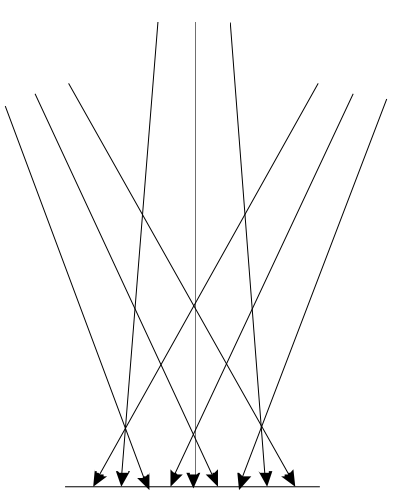

FIG. 7. Equivalent radial layout PLS, common image plane.

responds to the infinite position of a virtual camera producing an auxiliary image. For the case of PLS (Fig. 6), the partial projections are non-orthogonal ones which correspond to finite positions of virtual cameras (relative to the corresponding plane of a partial projection).

In Fig. 6a, b the local axes and projection rays bounding the field of view (FOV) are shown for each virtual camera of PLS case. Their intersection is the location of the camera. The basic difference between two variants Fig. $6 \mathrm{a}$ and $6 \mathrm{~b}$ is in the different focal distance of cameras.

This difference can be interpreted as a movement of PLS cameras to infinity along their axes with corresponding changes in the focal distance. The PLS picture corresponding to the longer focal distance (Fig. 6b) looks very similar to Fig. 5 for IP case. The closer the camera is to the infinity, the closer pictures in Fig. $6 \mathrm{~b}$ and Fig. 5 are. Therefore the infinite camera location can be also applied for PLS as a special case.

The picture of rays Fig. 6 is equivalent to the layout in which the period of elementary images is not equal 
to that of the lens array; see Fig. 7 where the image plane is shown.

The layout Fig. 7 seems to be principally possible in displaying since, it only requires different scaling of the image plane as compared to Fig. 4 with the same position of nodal points. Therefore it could be applied for both IP and PLS.

However this layout looks quite impossible in taking IP pictures because in this case it would require an increased inclination of the axes of the elementary lenses what is not a feature of a regular lens array. Probably, this could be simulated by using an irregular lens array with a non-plane, curved array. But this is only valid for very small angles (effectively non equal vertical displacements of lenses within the array) whereas the PLS angles are not principally limited.

\section{Parallel layout}

From the other hand, one can consider a parallel layout of cameras with a finite focal distance, widely used in PLS (Fig. 8).

This layout, if ever implemented for IP with using a lens array, would require something essentially unusual (paradoxical) features from a lens array. Namely, in this case, not only axes of lenses should be incrementally inclined like in Fig. 7 but also the rays passing the lenses should remain parallel, see Fig. 9 where an equivalent of Fig. 8 is shown.

The layout Fig. 9 can be interpreted as lenses of infinite focal distance looking in radial directions, from which images are projected non-orthogonally onto the same image plane. This does not seem to be possible to implement with a lens array, even if irregular. This makes the most widely used, parallel layout of PLS to be virtually impossible for IP.

\section{IMAGE CELLS. SIMULATION EXPERIMENTS}

In both groups images in the image plane are composed of cells of equal size. In the most cases the cells are squares (or rectangles) arranged in rows and columns but sometimes can be hexagonal or whichever else periodical shape fills the plane. The essential features of two groups are the following.

PLS. The source is the view images, obtained from regularly displaced camera(s). Generally, the camera displacement does not relate to the cell period. The view images can be processed pixel-wise so that the cell is composed from corresponding pixels of all view images $[9,10]$.

IP. The source is the elemental images, each next obtained under an elementary lens being in this case a small camera. The period of elementary lenses is equal to the cell period [3]. The source images can be pro-

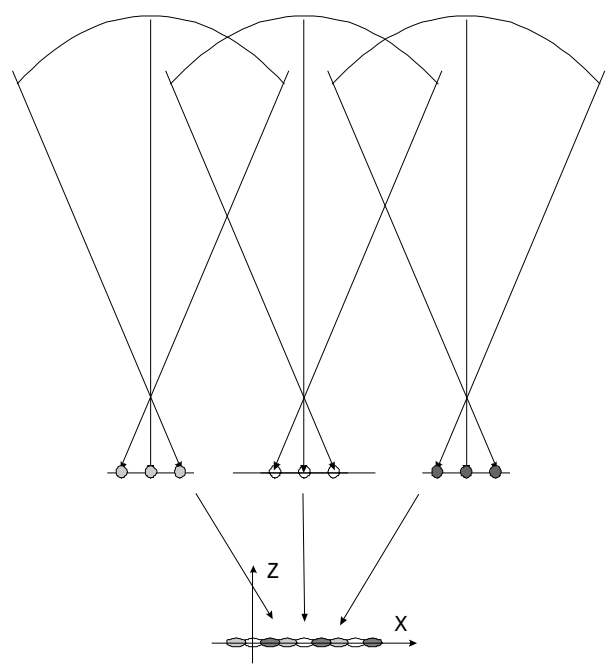

FIG. 8. Parallel layout PLS.

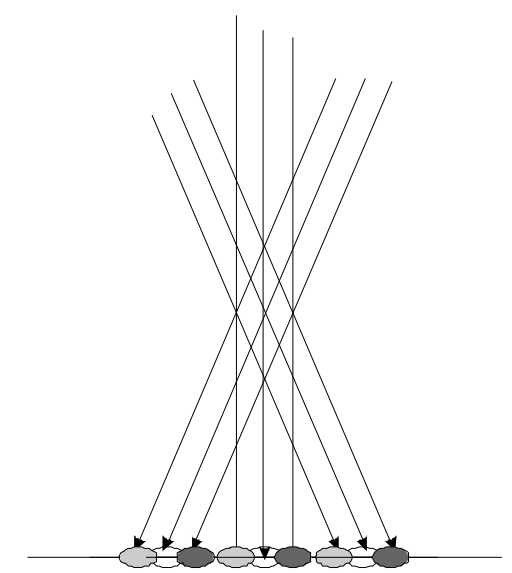

FIG. 9. Equivalent parallel layout PLS, common image plane.

cessed in order to increase the depth range [11] or to transform the pseudoscopic integral images into orthoscopic [12]. None of these procedures changes the basic layout of the elemental images each of them virtually corresponds to an elementary lens being a camera.

In both cases it seems to be reasonable to process images by cells, without crossing the inter-cell boundaries, if possible. The composed structure of cells can be determined, e.g., experimentally.

An experimental comparison of the two groups can be made, for example, by processing images of groups with using the same method. An image belonging to one group is processed with using methods of another. If the similarity is absent, i.e. groups do not relate to each other, the result will be unpredictable and will not look similar to any of source images. From the other hand, if the result would correspond to the original images of this or that group, the similarity would exist and the result will correspond to expectations based on another group. Since the image originally belongs to the first group, 


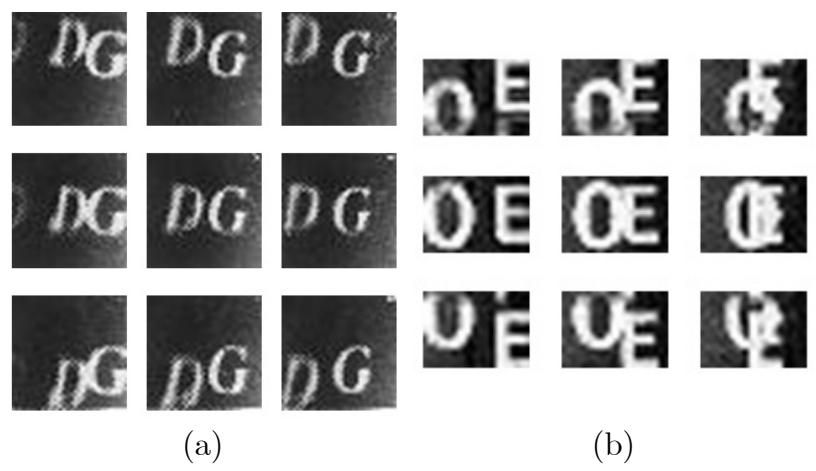

FIG. 10. Restored view images (a selection 3x3).

this would prove that the pixels layouts in both groups are the same or at least very close.

In order to do that we took several IP images randomly selected from one of the volumes of SPIE Proceedings devoted to 3D displaying. From this volume we only used the images unambiguously referred to as IP images in corresponding articles [12] and [13]. Both images contain letters, DG in the first case and $\mathrm{OE}$ in the second. These IP images are of different natures. The first image (Fig. 10a in [12]) is the processed orthoscopic integral image [12] while the second one (Fig 6 in [13]) is a computer-generated integral image [13]. With that, note that prior to processing we had to mirror the original image Fig. 10a [12]; otherwise it was not possible to obtain correct (non-mirrored) letters in a regular way.

Then, we used the image processing software created several years ago independently basing on the principles of PLS model $[9,10]$ which definitely corresponds to PLS technique but not IP. Note that our software was created several years ago especially to process PLS images. In the current experiments it was used in its original, untouched form.

The processing software was intended to compose a PLS image from the set of view images in accordance to PLS pixel layout. With that, there is an option in that software program which allows an inverse operation, decomposing a cellular composed PLS image into a matrix of view images. Also it is possible to process 2 geometrically different types of composed images, when the image plane is located in front of the plane of the light sources or behind it. These types differ in $180^{\circ}$ rotation of cells and correspond to two possible location of the image plane, behind and in front of PLS matrix. In this paper we used the decomposition option and non-rotated cells. Due to sampling conditions of our experiments, each cell consists of 20x20 pixels.

The results of processing IP images of different nature by an PLS algorithm are sets of elementary images arranged in the matrix 20x20, a selection $3 \times 3$ of which is shown in Fig. 10.

Shown in Fig. 10 are selections $3 \times 3$ of two restored
PLS view image sets $20 \times 20$ extracted from the original IP images. These were extracted from composed IP images by our PLS processing software. The selection includes the central view image and the view images near the edges of the restored total 20x20 matrix of images.

The "elemental" IP images can be seen within cells Fig. 10a in [12]. Also note that the central horizontal row of the restored PLS view image Fig. 10a appears to be very close to "observation by an observer" [12] but a difference in order of images anyway exists. A possible reason could be in the mentioned reflection applied before processing which implicitly causes the inversed order of restored images. Neither this tiny difference however does not affect the similarity, nor the different nature of IP images.

By analyzing the restored PLS view image sets Fig. 10 the relative locations of the restored objects can be found qualitatively after processing:

Screen, letter G, letter D, observer in the first case (Fig. 10a) and

Letter $\mathrm{O}$, screen, letter $\mathrm{E}$, observer in the second (Fig. 10b),

i.e. the letters $\mathrm{D}$ and $\mathrm{G}$ are located at the same side of the screen while the letters $\mathrm{O}$ and $\mathrm{E}$ are at two different sides. These locations which match the original locations of the letters (in depth) are the following. In the first case these are "two letters $\mathrm{O}$ and $\mathrm{E}$ formed at $20 \mathrm{~mm}$ in front and behind the pinhole array" [13], i.e. the letters are situated at both sides of a screen. And "the reference object plane is at $102.5 \mathrm{~mm}$. The letters are separated by $60 \mathrm{~mm}$ in depth" [12], i.e. the locations of letters are $72.5 \mathrm{~mm}$ and $132.5 \mathrm{~mm}$ at the same side of a screen.

In this paper we did not perform the depth calculations. Only the relative locations are found because in order to find the absolute ones we would need some more additional information.

\section{DISCUSSION}

Basing on variety of layouts in Section III one can say that the first stage (image capturing) provides relatively more freedom (less restrictions) in camera layouts than the second one, especially for PLS. The PLS is not limited however with it; more camera layouts are also possible [14] and PLS may include such camera layouts as parallel and radial with arbitrary focal distances of cameras (finite or infinite). The PLS allows more freedom in selecting cameras themselves (the focal length of a camera should not necessarily fit the focal length of a lenticular plate) as well as in their locations as mentioned above. Thus, the IP set of layouts can be considered as a subset of the possible layouts PLS.

The displaying stage can be typically implemented by using a lens array or its optical equivalents (say, a pin- 
hole array). In many cases we can control the cameras at the input stage of PLS individually whereas we cannot similarly control the lenses of a lens array at the displaying stage. In the simulation experiments, the restored locations are found for the parallel layout. It is mportant that the experiments were performed for IP images with using PLS technique. The IP images were of different nature (orthoscopic and computer-generated). These factors make the comparison more independent.

The layouts of one group can be considered as a subset of layouts of another group. The simulation experiments confirm that cells of the composed images are arranged similarly. Besides, the similarity in the viewing zones of both mentioned groups was already found [15]. Therefore one can consider both groups to be representatives of some more general 3D imaging technique, a cellular imaging (CI) the most important feature of which would be in a cellular structure of the image in the image plane.

In this case, the two groups, PLS and IP can be considered as particular cases of CI. The IP technique deals with elemental images obtained under each elementary lens. Any recording media has a finite resolution capacity. Therefore to make the elemental images to hold more content, IP needs bigger size of elementary lenses. To fulfill that, the IP image cell is typically $20 \times 20$ pixels or more. The PLS technique, on the other hand, requires many view images to be taken by camera(s) simultaneously. The total number of view images is $N^{2}$ where $\mathrm{N}$ is the number of camera positions in one dimension. From a practical point of view, it is not very convenient (or at least it is time consuming) to deal with $N^{2}$ view images of high resolution. This practical limitation makes the PLS image cell size smaller. Typically, the image cell of PLS includes about $6 \times 6$ pixels or less. This is the numerical difference between two groups.

Then, CI would deal with images build of cells. For digital images the size of a pixel is not an infinitesimal, and the cell boundary may not always fit the pixel boundary exactly but rather may lie somewhere within a pixel. This is perhaps a general case of CI processing and in order to avoid possible boundary effects and artifacts, the pixels located at cell boundaries should be processed separately in a different manner. A special situation happens when each cell consists of an integer number of pixels. In this case, the image processing has no side effects and is exact. All pixels within a cell are processed in the same manner. For an integer image cell, the perceptual visual image is more sharp and distinctive [16] and in this case we can say the pixel cell instead of the image cell.

\section{CONCLUSION}

A variety of layouts is classified and analyzed in terms of projections. Principally, the layouts may relate to any stage. However in practice, some of them can not be implemented in the second stage and therefore most of them actually relate to the first stage, capturing. For layouts with separate image planes, the corresponding equivalent layouts with the common plane are found providing a regular way to compare layouts. Also, the analytical formulas were found, in particularly for images with the equal displacement, as well as the transformation matrix.

Basing on relationship between sets of layouts one can say that IP can be treated as a particular case of the PLS, namely the radial layout of effective virtual cameras having the infinite focal distance (so as orthogonal projection is only in use). This confirms that an IP image virtually contains PLS view images for the parallel camera layout.

The simulation experiments based on the image processing were also performed for the second stage, displaying. The relative restored locations of objects coincide with the original relative locations of IP objects $[12,13]$. This indicates that it is possible to process IP images by using PLS technique and obtain reasonable results matching the original locations of IP objects, at least qualitatively. This has been demonstrated with a couple of randomly chosen IP images of different origin, and therefore one can consider these simulation experiments to be a direct experimental confirmation of similarity in question.

The layouts, analytical formulas, and experimental results show that for several layouts the images of both groups are similar whereas for other ones they are not. Therefore, the basic relationship between the basic layouts of two groups looks like follows: PLS is more general case of 3D imaging than IP and in few particular cases their layouts can match each other.

Basing on the similarity confirmed in this article, one may conclude that considering the cellular images in general would be useful for both IP and PLS. The theoretical and experimental consideration given in the current article may allow considering both groups, PLS and IP as representatives of more general 3D imaging technique, a cellular imaging $(\mathrm{CI})$. A cellular processing may require some special calculation techniques and this case should be considered separately.

\section{REFERENCES}

1. J.-Y. Son, V. Saveljev, D.-S. Kim, S.-K. Kim, and M.-C. Park, "Perceived images in IP and other multiview 3D imaging methods," Proc. SPIE 6016, 223-230 (2005).

2. B. Javidi and F. Okano, Eds., Three-Dimensional Television, Video, and Display Technologies (Springer, Germany, 2002).

3. F. E. Ives, "Color photography," U.S. patent 666424 (1901). 
4. J.-Y. Son, V. Saveljev, Y.-J. Choi, J.-E. Bahn, S.-K. Kim, and H.-H. Choi, "Parameters for designing autostereoscopic imaging systems based on lenticular, parallax barrier, and integral photography plates," Opt. Eng. 42, 33263333 (2003).

5. C. Wu, A. Aggoun, M. McCormick, and S. Y. Kung, "Depth extraction from unidirectional image using a modified multi-baseline technique," Proc. SPIE 4660, 135145 (2002).

6. J.-H. Park, S. Jung, H. Choi, Y. Kim, and B. Lee, "Depth extraction by use of a rectangular lens array and onedimensional elemental image modification," Appl. Opt. 43, 4882-4895 (2004).

7. H. S. M. Coxeter, Projective Geometry (Springer, USA, 2003), Chapter 12.

8. A. H. Watt, 3D Computer Graphics (Pearson Education Ltd., UK, 2000), Chapters 1, 5.

9. S.-S. Kim, K.-H. Sohn, V. Saveljev, E. F. Pen, J.-Y. Son, and J.-H. Chun, "A full parallax three-dimensional imaging system based on a point light source array," Jpn. J. Appl. Phys. 40, 4913-4915 (2001).

10. J.-Y. Son, V. V. Saveljev, Y.-J. Choi, and J.-E. Bahn, "Full parallax image generation," Proc. SPIE 4660, 116122 (2002).

11. H. Liao, M. Iwahara, Y. Katayama, N. Hata, and T.
Dohi, "Three-dimensional display with a long viewing distance by use of integral photography," Opt. Lett. 30, 613-615 (2005).

12. B. Javidi, R Martinez-Cuenza, G. Saavedra, and M. Martinez-Corral, "Orthoscopic, long-focal-depth integral imaging by hybrid method," Proc. SPIE 6392, 639203 (2006).

13. H.-J. Choi, Y.-H. Kim, S.-W. Cho, J.-H. Kim, Y.-M. Kim, and B.-H. Lee, "A thin 3D-2D convertible integral imaging system with a pinhole array on a polarizer," Proc. SPIE 6392, 639200 (2006).

14. H. Yamanoue, "The differences between toed-in camera configurations and parallel camera configurations in shooting stereoscopic images," in Proc. IEEE International Conference on Multimedia and Expo 2006 (ICME 2006) (Toronto, Ontario, Canada, July 2006), pp. 17011704 .

15. J.-Y. Son, V. Saveljev, K.-T. Kim, M.-C. Park, and S.-K. Kim, "Comparisons of perceived image in multiview and integral photography based three-dimensional imaging systems," Jpn. J. Appl. Phys. 46, 1057-1059 (2007).

16. J.-Y. Son, V. V. Saveljev, S.-H. Kim, and K.-H. Cha, "Viewing zones in non-integer type pixel cells," in Proc. 14th International Display Research Workshops (IDW'07) (Sapporo, Japan, Dec. 2007), pp. 2259-2262. 